\title{
Immune responses following third COVID-19 vaccination are reduced in patients with hematologic malignancies compared to patients with solid cancer
}

\section{Annika Fendler}

Cancer Dynamics Laboratory, The Francis Crick Institute

\section{Scott T.C. Shepherd}

Cancer Dynamics Laboratory, The Francis Crick Institute

\section{Lewis Au}

Cancer Dynamics Laboratory, The Francis Crick Institute

Katalin A. Wilkinson

Tuberculosis Laboratory, The Francis Crick Institute

\section{Mary Wu}

High Throughput Screening Laboratory, The Francis Crick Institute

Andreas M. Schmitt

Skin and Renal Units, The Royal Marsden NHS Foundation Trust

\section{Zayd Tippu}

Cancer Dynamics Laboratory, The Francis Crick Institute

\section{Sheima Farag}

Skin and Renal Units, The Royal Marsden NHS Foundation Trust

\section{Aljosja Rogiers}

Skin and Renal Units, The Royal Marsden NHS Foundation Trust

\section{Ruth Harvey}

Worldwide Influenza Centre, The Francis Crick Institute

\section{Eleanor Carlyle}

Skin and Renal Units, The Royal Marsden NHS Foundation Trust

\section{Kim Edmonds}

Skin and Renal Units, The Royal Marsden NHS Foundation TrustSkin and Renal Units, The Royal Marsden NHS Foundation Trust

\section{Lyra Del Rosario}

Skin and Renal Units, The Royal Marsden NHS Foundation Trust 
Skin and Renal Units, The Royal Marsden NHS Foundation Trust

\section{Mary Mangwende}

Skin and Renal Units, The Royal Marsden NHS Foundation Trust

\section{Lucy Holt}

Skin and Renal Units, The Royal Marsden NHS Foundation Trust

\section{Hamid Ahmod}

Skin and Renal Units, The Royal Marsden NHS Foundation Trust

\section{Justine Korteweg}

Skin and Renal Units, The Royal Marsden NHS Foundation Trust

\section{Tara Foley}

Skin and Renal Units, The Royal Marsden NHS Foundation Trust

\section{Taja Barber}

Cancer Dynamics Laboratory, The Francis Crick Institute

\section{Andrea Emslie-Henry}

Cancer Dynamics Laboratory, The Francis Crick Institute

\section{Niamh Caulfield-Lynch}

Cancer Dynamics Laboratory, The Francis Crick Institute

\section{Fiona Byrne}

Cancer Dynamics Laboratory, The Francis Crick Institute

\section{Benjamin Shum}

Cancer Dynamics Laboratory, The Francis Crick Institute

\section{Camille L. Gerard}

Cancer Dynamics Laboratory, The Francis Crick Institute

\section{Daqi Deng}

Cancer Dynamics Laboratory, The Francis Crick Institute

\section{Svend Kjaer}

Structural Biology of Disease Processes Laboratory, The Francis Crick Institute, London

\section{Ok-Ryul Song}

High Throughput Screening Laboratory, The Francis Crick Institute

\section{Christophe Queval}

High Throughput Screening Laboratory, The Francis Crick Institute

\section{Caitlin Kavanagh}

High Throughput Screening Laboratory, The Francis Crick Institute

\section{Emma C. Wall}

University College London Hospitals NHS Foundation Trust Biomedical Research Centre

\section{Edward J. Carr}

Cell Biology of Infection Laboratory, The Francis Crick Institute

\section{Sina Namjou}

Safety, Health \& Sustainability, The Francis Crick Institute 


\section{Simon Caidan}

Safety, Health \& Sustainability, The Francis Crick Institute

\section{Mike Gavrielidis}

Scientific Computing Scientific Technology Platform, The Francis Crick Institute James McRae

Metabolomics Scientific Technology Platform, The Francis Crick Institute

\section{Gavin Kelly}

Department of Bioinformatics and Biostatistics, The Francis Crick Institute

\section{Kema Peat}

Skin and Renal Units, The Royal Marsden NHS Foundation Trust

\section{Denise Kelly}

Skin and Renal Units, The Royal Marsden NHS Foundation Trust

\section{Aida Murra}

Skin and Renal Units, The Royal Marsden NHS Foundation Trust

\section{Kayleigh Kelly}

Skin and Renal Units, The Royal Marsden NHS Foundation Trust

\section{Molly O'Flaherty}

Skin and Renal Units, The Royal Marsden NHS Foundation Trust

\section{Robyn L. Shea}

Department of Pathology, The Royal Marsden NHS Foundation Trust

\section{Gail Gardner}

Translational Cancer Biochemistry Laboratory, The Institute of Cancer Research

\section{Darren Murray}

Translational Cancer Biochemistry Laboratory, The Institute of Cancer Research

\section{Nadia Yousaf}

Lung Unit, The Royal Marsden NHS Foundation Trust

\section{Shaman Jhanji}

Anaesthetics, Perioperative Medicine and Pain Department, The Royal Marsden NHS Foundation Trust, London

\section{Nicholas Van As}

Clincal Oncology Unit, The Royal Marsden NHS Foundation Trust

\section{Kate Young}

Skin and Renal Units, The Royal Marsden NHS Foundation Trust

Andre J.S. Furness

Skin and Renal Units, The Royal Marsden NHS Foundation Trust

\section{Lisa Pickering}

Skin and Renal Units, The Royal Marsden NHS Foundation Trust

\section{Rupert Beale}

Cell Biology of Infection Laboratory, The Francis Crick Institute 


\section{Charles Swanton}

Cancer Evolution and Genome Instability Laboratory, The Francis Crick Institute

\section{Sonia Ghandi}

Neurodegeneration Biology Laboratory, The Francis Crick Institute

\section{Steve Gamblin}

Structural Biology of Disease Processes Laboratory, The Francis Crick Institute

\section{David L.V. Bauer}

RNA Virus Replication Laboratory, The Francis Crick Institute,

\section{George Kassiotis}

Retroviral Immunology Laboratory, The Francis Crick Institute

\section{Michael Howell}

High Throughput Screening Laboratory, The Francis Crick Institute

\section{Emma Nicholson}

Haemato-oncology Unit, The Royal Marsden NHS Foundation Trust

\section{Robert J. Wilkinson}

Tuberculosis Laboratory, The Francis Crick Institute

\section{James Larkin}

Skin and Renal Units, The Royal Marsden NHS Foundation Trust

\section{Samra Turajlic ( $\square$ samra.turajlic@crick.ac.uk)}

Cancer Dynamics Laboratory, The Francis Crick Institute

\section{Research Article}

Keywords: COVID-19, solid cancer, hematologic cancer, vaccine, booster

Posted Date: December 22nd, 2021

DOI: https://doi.org/10.21203/rs.3.rs-1191603/v1

License: (c) (1) This work is licensed under a Creative Commons Attribution 4.0 International License. Read Full License 


\section{Abstract}

Not all patients with cancer, in particular those with hematogic malignancies, develop functional immunity against SARS-CoV-2 variants of concern (VOC) following COVID-19 vaccines. Durability of vaccine-induced immunity after two doses and the impact of a third dose were evaluated in CAPTURE (NCT03226886), a longitudinal prospective cohort study of vaccine responses in patients with cancer. In evaluating 316 patients, at a median of 111 days following two doses of either BNT16b2 or ChadOX, we observed a time-dependant decline in neutralising antibody titres (NAbT) in a proportion of patients, where NAbTs became undetectable against Delta and Beta in $17 \%$ and $15 \%$ of patients, respectively. Vaccine-induced $T$ cell responses declined in $44 \%$ of patients. Patients with breakthrough infections following two vaccines doses were characterised by absent/low NAbT to Delta prior to infection. Administration of the third vaccine dose boosted NAb responses against VOC in the majority of patients with cancer, especially those with solid cancer. In patients with hematologic malignancies who had undetectable NAbT against Delta after two vaccine doses, 54\% did not develop NAb against both Beta and Delta following the third dose. Third vaccine dose boosted $T$ cell responses were boosted in patients with both solid and hematologic malignancies. These results provide critical information on vaccine responses in patients with cancer, especially against VOCs and support widespread access to a third COVID-19 vaccination in this patient group.

\section{Full Text}

Patients with cancer are at higher risk of severe COVID-19 (Grivas et al., 2021; Kuderer et al., 2020), and are currently prioritized for a third COVID-19 vaccine dose globally. Humoral and cellular immune responses are detected after two primary COVID-19 vaccine doses in most patients with cancer (Ehmsen et al., 2021; Fendler et al., 2021; Oosting et al.), although neutralising responses are reduced against variants of concern (VOC). Neutralising responses are frequently impaired in patients with hematologic malignancies especially those receiving B cell-depleting therapies (Ehmsen et al., 2021; Fendler et al., 2021; Thakkar et al.). As neutralising antibody (NAb) responses directly associate with vaccine efficacy (Gilbert Peter et al.; Khoury et al., 2021), these patients without a neutralising response are at higher risk of breakthrough infections (Hippisley-Cox et al., 2021). Here, we report follow-up results from CAPTURE (NCT03226886), a longitudinal, prospective cohort study of vaccine response in patients with cancer, relative to the duration of response after two doses of either BNT162b2 (Pfizer) or ChAdOx1 (AstraZeneca); and following third vaccination with BNT162b2. We present data on NAb and T cell responses against whole live virus, including wildtype SARS-CoV-2 (WT), Beta, and Delta VOC. We specifically evaluated responses to Beta and Delta given their known immune-evasive capacity.

We assessed the durability of NAb responses in 353 patients [77\% $(n=271)$ with solid, and $23 \%(n=82)$ with hematologic malignancies], (Table S1), following two doses of COVID-19 vaccine [72\% $(n=255)$ ChAdOx1, 28\% ( $\mathrm{n}=98)$ BNT162b2]. NAbs against WT SARS-CoV-2 were undetectable 14-28 days and up to 110 days (range $84-153)$ following the second dose in $4 \%(n=12)$ of patients with solid, and $30 \%$ $(n=25)$ of patients with hematologic malignancies. In those who initially had detectable post-second dose 
NAb against WT ( $n=316 ; 71 \%$ against Beta, and $62 \%$ against Delta), we observed a time-dependent decline in NAb titres (NAbT) during follow up (median of 111 days, range: 37-252 day after the second vaccine dose) (Figure S1A). After an initial response to two vaccine doses, in patients with solid cancer $(n=259), 1 \%(n=3)$ had undetectable NAbs against WT, 17\% $(n=43)$ against Beta, and 18\% $(n=47)$ against Delta; in patients with hematologic malignancies $(n=57), 7 \%(n=4)$ had undetectable NAbT against WT, $9 \%(n=5)$ against Beta, and 16\% $(n=9)$ against Delta. The proportions of those with waning NAb did not differ significantly between patients with solid or hematologic malignancies, apart from WT $\left(\mathrm{Chi}^{2}\right.$ test: WT, $p=0.02$, Beta, $p=0.16$, Delta, $p=0.67$ ).

We previously reported that T cell responses, measured 14-28 days after the second dose, are comparable between patients with solid and hematologic malignancies and can also be detected in those without NAb responses (Fendler et al., 2021). During follow up [median of 93 days (range: 63-171 days) after the second dose], we evaluated T cell responses in 55 patients. Patients without a detectable $T$ cell response following the second dose remained negative. In those with an initial response $(n=43$ with solid and $n=12$ with hematologic malignancies) (Figure 1SB), it was maintained in $49 \%(n=21)$ of patients with solid and $42 \%(n=5)$ with hematologic malignancies (Wilcoxon signed rank test, $p=0.56)$.

During the course of routine clinical care, 8 CAPTURE participants $(n=7$ with solid and $n=1$ with hematological malignancy) were diagnosed with SARS-COV-2 following two vaccine doses between July and October 2021 [median time between second vaccine dose and infection: 118 days (range: 59-173)], likely to be caused by the Delta variant that was dominant in the U.K. at that time. The symptoms were either mild [ $n=7$ patients; WHO severity score 2-3; fever $(n=5)$, coryza $(n=4)$, anosmia $(n=4)$ and cough $(n=3)]$ or absent $(n=1$ patient), not requiring hospital care, and all patients recovered. We evaluated immune responses prior to infection following two vaccine doses; while all patients had detectable NAbT against WT SARS-CoV-2, only one had detectable NAbT against Delta. Following infection, all patients mounted a detectable neutralising response to Delta (Figure S1C). T cell responses were evaluable in 5 patients prior to infection and in 7 patients following infection. While only $1 / 5$ patients had detectable $T$ cell responses to WT prior to infection, $5 / 7$ had a detectable $T$ cell response following infection, including 2/4 patients that had undetectable T cell responses before infection (Figure S1D).

We next evaluated 199 cancer patients [n=115 (58\%) with solid cancer] who received a third vaccine dose per UK guidelines. Patients who tested positive for SARS-CoV-2 by RT-PCR between second and third dose were excluded from this analysis. All patients received a third dose of BNT162b2, following two doses of either BNT162b2 (33\%) or ChAdOx1 (67\%) (Table S1). The median time between second and third vaccine dose was 176 days (range 65-274 days), and immune responses were measured at a median of 23 days after the third dose (range: 11-47 days). Prior to third dose, $88 \%(n=176)$ had detectable NAb against WT, but given the dominance of Delta, we considered all patients with undetectable NAb against Delta as 'non-responders' to two doses [51\% ( $n=102) ; 43 \%(n=50)$ of solid cancer and $62 \%(n=52)$ of hematologic malignancy] (Table S1). 
Considering non-responders to Delta after two vaccine doses, in solid cancer patients $(n=50)$, following the third dose $94 \%(n=47)$ had detectable NAb against Delta, and 88\% ( $n=44)$ against Beta (Figure S1E); in patients with hematologic malignancy $(n=52)$, following the third dose $54 \%(n=28)$ had detectable NAb against Delta, and $54 \%(n=28)$ against Beta. The proportion of those with detectable NAb after third dose was significantly higher in patients with solid malignancies (Chi ${ }^{2}$ test: Beta; $p=0.0002$, Delta; $p<0.0001$ ). Finally, following the third dose we observed an increase in median NAbT against all variants in initial responders (patients with detectable NAbs against Delta after two vaccine doses).

Our data indicate that a third vaccine dose can generate NAb in patients who are non-responders following two doses, and further boosts NAbT against VOCs in responders. However, the proportion of patients with hematologic malignancies with undetectable NAbT against Delta following the third vaccine dose remains significant (46\%).

Multivariable binary regression analysis shows that the presence of hematologic malignancy was significantly associated with undetectable NAb against Beta or Delta after the third dose (variables included: cancer type, age, primary vaccine type, and sex). Considering patients with hematologic malignancies in a further multivariate analysis (see Table S1 for included variables), primary vaccination with BNT162b2 ( $n=17)$ vs ChAdOx1 $(n=35)$ was significantly associated with lack of neutralising responses against Beta and Delta [BNT162b2: Delta 29\% $(n=5)$, Beta, 35\% $(n=6)$; ChAdOx1: Delta, 66\% $(n=23) ;$ Beta, $63 \%(n=22)]$, suggesting benefit of the heterologous vaccination approach. Following the third vaccine dose, in patients who had received anti-CD20 in the 12 months prior to the first vaccine dose, $1 / 6$ had detectable NAbT against Delta and 2/ 6 against Beta. In the group of patients who commenced anti-CD20 therapy between the second and third dose $3 / 4$ patients had detectable NAbT against Delta and Beta following the third dose.

Following stimulation with WT, Beta, and Delta spike-specific peptide pools T cell responses were measured by IFN-y ELISPOT (Fendler et al., 2021) in a subset of 48 patients [69\% $(n=33)$ solid cancer] representative of the cohort that received a third dose. Prior to third dose, $33 \%(n=11)$ of patients with solid and $40 \%(n=6)$ of patients with hematologic malignancies had detectable T cell responses. Following the third vaccine dose, this rose to $73 \%(n=24)$ and $73 \%(n=11)$, respectively. The number of $\mathrm{SFU} / 10^{6}$ was significantly increased after the third dose relative to post-second dose (Figure S1F).

Our data show a third dose of COVID-19 vaccine boosts NAb responses in patients with cancer including those that had undetectable NAbT following two vaccine doses, or where NAbT waned. We found that NAbT were higher in patients who received two doses of ChAdOx1 and a third dose of BNT162b2 compared to three doses of BNT162b2. Further, we show that T cell responses are amplified following third vaccine dose likely offering additional protection especially in individuals with low or absent neutralising responses. Encouragingly, the proportion of patients with solid cancer that had detectable responses after third vaccination is high and comparable to individuals without cancer. In contrast, a substantial proportion of patients with hematologic malignancies still had undetectable neutralising responses following third vaccine dose, especially against VOCs and may remain at risk of breakthrough 
infection. These findings are particularly pertinent given reports of reduced vaccine efficacy and NAb activity against the emerging Omicron VOC compared to Delta (Cele et al., 2021).

\section{Declarations}

Declaration of interests

The authors declare no competing interests.

\section{References}

1. Cele, S., Jackson, L., Khan, K., Khoury, D.S., Moyo-Gwete, T., Tegally, H., Scheepers, C., Amoako, D., Karim, F., Bernstein, M., et al. (2021). SARS-CoV-2 Omicron has extensive but incomplete escape of Pfizer BNT162b2 elicited neutralization and requires ACE2 for infection. medRxiv, 2021.2012.2008.21267417.

2. Ehmsen, S., Asmussen, A., Jeppesen, S.S., Nilsson, A.C., Østerlev, S., Vestergaard, H., Justesen, U.S., Johansen, I.S., Frederiksen, H., and Ditzel, H.J. (2021). Antibody and T cell immune responses following mRNA COVID-19 vaccination in patients with cancer. Cancer Cell 39, 1034-1036.

3. Fendler, A., Shepherd, S.T.C., Au, L., Wilkinson, K.A., Wu, M., Byrne, F., Cerrone, M., Schmitt, A.M., Joharatnam-Hogan, N., Shum, B., et al. (2021). Adaptive immunity and neutralizing antibodies against SARS-CoV-2 variants of concern following vaccination in patients with cancer: the CAPTURE study. Nature Cancer.

4. Gilbert Peter, B., Montefiori David, C., McDermott Adrian, B., Fong, Y., Benkeser, D., Deng, W., Zhou, H., Houchens Christopher, R., Martins, K., Jayashankar, L., et al. Immune correlates analysis of the mRNA-1273 COVID-19 vaccine efficacy clinical trial. Science 0 , eab3435.

5. Grivas, P., Khaki, A.R., Wise-Draper, T.M., French, B., Hennessy, C., Hsu, C.Y., Shyr, Y., Li, X., Choueiri, T.K., Painter, C.A., et al. (2021). Association of clinical factors and recent anticancer therapy with COVID-19 severity among patients with cancer: a report from the COVID-19 and Cancer Consortium. Annals of Oncology 32, 787-800.

6. Hippisley-Cox, J., Coupland, C.A., Mehta, N., Keogh, R.H., Diaz-Ordaz, K., Khunti, K., Lyons, R.A., Kee, F., Sheikh, A., Rahman, S., et al. (2021). Risk prediction of covid-19 related death and hospital admission in adults after covid-19 vaccination: national prospective cohort study. BMJ 374, n2244.

7. Khoury, D.S., Cromer, D., Reynaldi, A., Schlub, T.E., Wheatley, A.K., Juno, J.A., Subbarao, K., Kent, S.J., Triccas, J.A., and Davenport, M.P. (2021). Neutralizing antibody levels are highly predictive of immune protection from symptomatic SARS-CoV-2 infection. Nature Medicine 27, 1205-1211.

8. Kuderer, N.M., Choueiri, T.K., Shah, D.P., Shyr, Y., Rubinstein, S.M., Rivera, D.R., Shete, S., Hsu, C.Y., Desai, A., de Lima Lopes, G., Jr., et al. (2020). Clinical impact of COVID-19 on patients with cancer (CCC19): a cohort study. Lancet 395, 1907-1918. 
9. Oosting, S.F., van der Veldt, A.A.M., GeurtsvanKessel, C.H., Fehrmann, R.S.N., van Binnendijk, R.S., Dingemans, A.-M.C., Smit, E.F., Hiltermann, T.J.N., den Hartog, G., Jalving, M., et al. mRNA-1273 COVID-19 vaccination in patients receiving chemotherapy, immunotherapy, or chemoimmunotherapy for solid tumours: a prospective, multicentre, non-inferiority trial. The Lancet Oncology.

10. Thakkar, A., Gonzalez-Lugo, J.D., Goradia, N., Gali, R., Shapiro, L.C., Pradhan, K., Rahman, S., Kim, S.Y., Ko, B., Sica, R.A., et al. Seroconversion rates following COVID-19 vaccination amongst patients with cancer. Cancer Cell.

\section{Supplementary Files}

This is a list of supplementary files associated with this preprint. Click to download.

- 20211218SupplementaryDataFileTurajlicclean.pdf 